\title{
CUIDADOS PALIATIVOS: VISÃO DE ENFERMEIROS DE UM HOSPITAL DE ENSINO
}

Mariana Carneiro de Oliveira ${ }^{1}$, Francine Lima Gelbcke², Luciana Martins da Rosa ${ }^{3}$, Mara Ambrosina De Oliveira Vargas ${ }^{4}$, Juliana Balbinot Girondi Reis ${ }^{5}$

Objetivo: identificar se há a inserção do conceito e dos princípios dos Cuidados Paliativos definidos pela Organização Mundial de Saúde na atuação de enfermeiros de Unidades de Clínicas Médicas e da Comissão de Cuidados Paliativos e Controle da Dor de um HospitalEscola da Região Sul do Brasil. Metodologia: pesquisa qualitativa com coleta de dados mediante entrevista semiestruturada com 22 enfermeiros, submetidas à técnica de análise de conteúdo. Resultados: o enfermeiro vincula os Cuidados Paliativos com o processo de morte dos pacientes. A comunicação limitada oblitera as condutas tomadas pelos membros da equipe. As pessoas com doença crônica são encaminhadas tardiamente, submetendo-se a ações reducionistas que não proporcionam qualidade de vida. Conclusões: os princípios da filosofia dos Cuidados Paliativos estão inseridos parcialmente na prática dos profissionais. Há demanda de formação acadêmica e em serviço.

Descritores: Cuidados Paliativos, Doença Crônica, Cuidados de Enfermagem, Serviço Hospitalar de Enfermagem.

\section{PALLIATIVE CARE: NURSES VISION OF A TEACHING HOSPITAL}

Objective: identify if there is the inclusion of the concept and the principles of palliative care as defined by the World Health Organization in the performance of Medical Clinical Unit nurses and the Committee on Palliative Care and Pain Management of a teaching hospital in southern Brazil. Methodology: qualitative research with data collection through semi-structured interviews with 22 nurses, submitted to the technique of content analysis. Results: the nurse links Palliative Care of death of patients process. The limited communication obliterates the steps taken by team members. People with chronic disease are referred late, undergoing reductionist actions that do not provide quality of life. Conclusion: the principles of the philosophy of palliative care are partially inserted in professional practice. There is demand for academic training and service.

Descriptors: Palliative Care, Chronic Disease, Nursing Care, Nursing Service Hospital.

\section{CUIDADOS PALIATIVOS: VISIÓN DE ENFERMEROS DE UN HOSPITAL UNIVERSITARIO}

Objetivo: identificar si existe la inclusión del concepto y los principios de los cuidados paliativos según lo definido por la Organización Mundial de la Salud en el desempeño de médicos enfermeras Clinical Unit y el Comité de Cuidados Paliativos y Tratamiento del Dolor de un hospital universitario en el sur de Brasil. Metodología: la investigación cualitativa con la recolección de datos a través de entrevistas semiestructuradas con 22 enfermeras, sometidos a la técnica de análisis de contenido. Resultados: la enfermera une cuidados paliativos de la muerte del proceso de los pacientes. La comunicación limitada borra las medidas adoptadas por los miembros del equipo. Las personas con enfermedades crónicas se refieren tarde, sometidos a acciones reduccionistas que no proporcionan la calidad de vida. Conclusións: los principios de la filosofía de los cuidados paliativos se insertan parcialmente en la práctica profesional. Hay demanda de formación académica y de servicio.

Descriptores: Cuidados Paliativos, Enfermedad Crónica, Atención de Enfermería, Servicio de Enfermería en Hospital.

1Enfermeira. Mestrado Profissional Gestão do Cuidado de Enfermagem da Universidade Federal de Santa Catarina (UFSC).

${ }^{2}$ Enfermeira. Doutora em Enfermagem. Docente do Departamento de Enfermagem e do Programa de Pós Graduação Enfermagem UFSC.

${ }^{3}$ Enfermeira. Doutora em Enfermagem. Docente do Departamento de Enfermagem e do Curso de Mestrado Profissional Gestão do Cuidado de Enfermagem da UFSC. E-mail: luciana.m.rosa@ufsc.br.

${ }^{4}$ Enfermeira. Doutora em Enfermagem. Docente do Departamento de Enfermagem e do Programa de Pós Graduação Enfermagem UFSC

5Enfermeira. Doutora em Enfermagem. Docente do Departamento de Enfermagem e do Curso de Mestrado Profissional Gestão do Cuidado de Enfermagem da UFSC. 


\section{INTRODUÇÃO}

Na busca por uma assistência de qualidade, em 2002, a Organização Mundial de Saúde (OMS) conceituou que os Cuidados Paliativos (CP) abrangem os pacientes e seus familiares que convivem com doenças que ameaçavam a vida desde o seu diagnóstico, entendendo que, muitos problemas que aparecem na fase final da doença, poderiam ser melhor controlados ou cessados antes dessa etapa ${ }^{(1)}$. Além disto, ampliou-se a ideia de cuidado, agora entendendo a patologia como um processo, que afeta o portador ao longo de sua vida e da convivência com a doença (2). Logo, o que caracteriza a indicação de CP ao portador de uma doença crônica é seu grau de sofrimento e não a probabilidade de morte, pois buscam o alivio de sintomas ${ }^{(2-4)}$. Na perspectiva dos $\mathrm{CP}$, sempre há algo para se fazer; assim, a expressão "fora de possibilidades terapêuticas" deve ser substituída por "fora da possibilidade de tratamento modificador da doença", ampliando, dessa forma, a visão dos $\mathrm{CP}^{(2)}$.

A prática dos $\mathrm{CP}$ não se baseia em protocolos estanques, mas em princípios, determinando a visão de filosofia de assistência pautada em: 1) aliviar a dor e os sintomas considerados estressantes pelo paciente; 2) afirmar a vida e considerar a morte como um processo natural; 3) não acelerar e nem postergar a morte; 4) integrar aspectos psicológicos e espirituais no cuidado; 5) oferecer autonomia ao paciente; 6) oferecer suporte para auxiliar os familiares a compreenderem a evolução da doença e se sentirem amparados no momento do luto; 7) assistir de maneira multiprofissional; 8) iniciar seus princípios juntos com medidas curativas logo que se diagnostique uma doença ameaçadora da vida ${ }^{(2-4)}$.

Nessa perspectiva, ao ser implantada uma Comissão de CP em um Hospital-Escola da Região Sul do Brasil, emergiram muitas dúvidas na equipe de enfermagem. Logo, constatou-se a prioridade em promover uma discussão mais ampla do conceito e dos princípios que alicerçam os CPs e de quais pacientes se inserem na lógica desses princípios. Ainda, na prática, os profissionais observavam que várias medicações passavam a ser suspensas da prescrição, pela Comissão de $\mathrm{CP}$, sem maiores esclarecimentos aos enfermeiros, familiares e equipe. Também observavam que a Comissão registrava no prontuário que determinado paciente encontrava-se em CP, sempre vinculado à noção de que o paciente estava em processo de morte imediata.

Frente a tais inquietações, buscou-se, neste estudo, identificar se há a inserção do conceito e dos princípios dos CP definidos pela OMS na atuação de enfermeiros das Clínicas Médicas e da Comissão de CP e Controle da Dor de um Hospital-Escola da Região Sul do Brasil.

\section{METODOLOGIA}

Pesquisa qualitativa, realizada em um Hospital-Escola da Região Sul do Brasil, com 22 enfermeiros que atuavam nas Clínicas Médicas e na Comissão de CP e Controle da Dor. A coleta de dados foi realizada no mês de maio de 2013, através de entrevistas semiestruturadas gravadas, em sala reservada, e durante o turno de serviço de cada participante do estudo. As entrevistas transcritas foram enviadas via correio eletrônico para validação de cada participante do estudo. Os resultados foram submetidos à técnica de Análise de Conteúdo. A sustentação teórica foi direcionada pelo conceito e princípios dos CP estabelecidos pela OMS. O estudo foi aprovado pelo Comitê de Ética da Universidade Federal de Santa Catarina, sob o parecer 253.280/2013.

\section{RESULTADOS}

A codificação das informações levou à definição de unidades de significação, agrupadas na categoria: Cuidados Paliativos: o significado e as limitações atribuídas por enfermeiros.

\section{Cuidados Paliativos: o significado e as limitações atribuídas por enfermeiros}

Foi constatado que o enfermeiro estabelece relação dos $\mathrm{CP}$ com a finitude dos pacientes e que este termo remete ao profissional o processo de morrer.

Diante disto, estabeleceu-se a seguinte unidade de significação: Cuidados Paliativos: finitude: 0 Cuidados Paliativos é o cuidado, o bem-estar do paciente na hora final. (E 15). Cuidados Paliativos já é o pré-óbito. (E 20). Cuidados Paliativos é o fim de um periodo muito grande de sofrimento. (E I).

Os relatos mostraram as dificuldades dos profissionais em aceitar a morte como um processo e que este vínculo está diretamente relacionado à falta de discussões sobre o assunto durante a formação acadêmica da equipe multiprofissional, visibilizados na unidade de significação: Cuidados Paliativos: carência de formação: Discutimos pouco, durante a nossa formação sobre a morte. Somos formados para salvar vidas, para curar. (E 16). Os Cuidados Paliativos foram jogados na Instituição e não houve preparo da equipe, não houve explicação do que era. (E 7).

Quando se questionou sobre a assistência ao portador de doença crônica sob a ótica dos CP, entre os fatores apontados, está a própria percepção do que são e para quem é indicado. Assim, estabeleceu-se a unidade de significação: Cuidados Paliativos: indicado para quem?: Eles decidem que é Cuidados Paliativos, então cortam a metade da prescrição, deixam as medicações para alivio da dor e a equipe de 
enfermagem pensa que é menos trabalho quando não se mexe mais, não come, não fala e não reclama, não tem que dar explicações, porque muitas vezes a familia já entendeu e acha que o que você está fazendo é por compaixão e além da sua obrigação. (E 19)

Apesar de considerarem os CP e a morte como conceitos muito próximos, os participantes apontaram em suas definições que os CP estão relacionados com aspectos além da morte, aproximando-se dos principios trazidos pela OMS. E, dentre as definições, o alívio dos sintomas apareceu com maior frequência. Assim, estabeleceu-se a unidade de significação intitulada: Cuidados Paliativos: alívio dos sintomas: Os Cuidados Paliativos trouxeram essa preocupação com o conforto, que antes não havia. (E 4). Acho que ele tenha uma qualidade de vida melhor. (E 22).

No entanto, o discurso acerca do conforto traz em seu bojo a percepção deste como algo próprio da Enfermagem, pois ao se questionar qual seria a diferença entre a prática de cuidado de enfermagem com a prática do $\mathrm{CP}$, alguns expressaram que o CP é um ramo da enfermagem: Para mim é um conceito de cuidado, a gente sempre fez isso. A gente sempre proporciona conforto ao paciente independente se para a equipe médica o paciente está fora das possibilidades de cura ou não. (E 7).

Os participantes relataram que as falhas na comunicação dificultam as condutas tomadas pelos membros da equipe multidisciplinar, principalmente quando depende de procedimentos médicos para que os demais cuidados aconteçam, como exemplo, melhora da analgesia. A partir dos achados abrangendo essa questão, estabeleceu-se a unidade de significação: Cuidados Paliativos: dificuldades na comunicação da equipe multiprofissional: A equipe médica demora muito para decidir o prognóstico do paciente, isso vai nos deixando angustiadas, principalmente para nós que estamos na linha de frente das dúvidas. A gente precisa urgentemente de discussões multiprofissionais e depois cada um com suas equipes, para que todos cresçam e evite fofocas e intrigas. (E 16).

\section{DISCUSSÃO}

O vínculo dos CP com a finitude, sinalizado nos relatos dos enfermeiros é, na maioria dos casos, associado à existência de uma formação deficitária nos currículos acadêmicos, principalmente, no que tange à comunicação e ao alivio dos sintomas, como a dor. Nesta direção, sinaliza-se que em países onde o desenvolvimento dos CP está enraizado no sistema de saúde, os currículos acadêmicos incluem o aprendizado do tema. No Brasil, evidencia-se uma lacuna, destacadamente nos cursos de Medicina e Enfermagem, pois o aprendizado sobre CP são tangenciados em abordagens como a morte, dor ou oncologia ${ }^{(5)}$.

Os depoimentos dos enfermeiros destacam a necessidade e importância da formação em CP e, além disso, refletem a atuação contraditória da equipe. De um lado, ela define que o paciente esteja em CP, quando a morte é esperada para horas e/ou dias, entretanto, ainda são instituídas medidas que causam desconforto, como exames e procedimentos invasivos, o que reforça a necessidade de formação e o entendimento do que é distanásia.

Diferentes estudos ${ }^{(6,7)}$ retratam condições para a qualidade do cuidado ligadas às habilidades pessoais. É destacada a necessidade na formação de enfermeiros com conhecimento e habilidades para cuidar de pessoas no final de suas vidas. O despreparo dos profissionais das mais diversas áreas em lidar com situações iminentes e irreversiveis de morte revela a morte e o processo do morrer como temas substancialmente pouco estudados durante - período de graduação. Em contraponto, está a necessidade de manter-se estável e integro em âmbito de atuação, através do cuidado aos seres humanos e não apenas através da objetivação de $\operatorname{cura}^{(8)}$.

Ainda quanto à necessidade de formação, é notória a necessidade urgente de uma revisão dos Projetos Pedagógicos dos Cursos de formação superior da área da saúde pra que possam educar para o cuidado específico dos doentes crônicos e para que cada futuro profissional compreenda o que significa CP e como devem ser realizados. Nesse sentido, sinaliza-se que os provedores de cuidados de saúde - assistentes sociais, enfermeiros, farmacêuticos, médicos, e outros - aprendem a cuidar bem, de um modo especial. Entre-tanto, todos devem saber como prestar bons $\mathrm{CP}$, o que pode reduzir significativamente o tempo de internação hospitalar ${ }^{(9)}$.

A carência de formação dos profissionais atuantes no cenário do estudo interfere, ainda, na indicação de quem 
deve ser encaminhado para os CP. Os relatos mostraram claramente que as pessoas com doença crônica são encaminhadas tardiamente, e, quando o são, realizam-se ações reducionistas que não proporcionam qualidade de vida.

Logo, a necessidade de melhorar o atendimento a pessoas com doenças crônicas, complexas, potencialmente fatais ou limitativas de vida é inquestionável ${ }^{(10)}$. Nessa direção, estudo evidencia que, na comparação entre CP prestados em ambientes institucionais e domiciliares, os membros da família ficam significativamente mais satisfeitos com o fim da vida de entes queridos acompanhados em unidades especializadas em CP. Ao focalizar-se na percepção de familiares, o estudo destaca a falta de instrumentos para medir a eficácia destes cuidados e a importância dessa mensuração para o desenvolvimento de cuidados de alta qualidade em locais onde novos modelos de assistência estão sendo desenvolvidos(11). $\mathrm{Na}$ verdade, o que está em questão é justamente que os profissionais que atuam em unidades especializadas em $\mathrm{CP}$, diferentemente dos profissionais que atuam em unidades hospitalares que cuidam tanto de pessoas que necessitam de CP como de pessoas que não demandam $\mathrm{CP}$, tendem a buscar subsidios, capacitando-se para desenvolver o melhor cuidado.

Os relatos dos participantes ainda destacaram o cuidado que possibilita conforto. Para os profissionais, a Enfermagem e os CP estão intimamente ligados aos princípios do cuidado de Enfermagem.

Porém, aponta-se que existe diferença entre um e outro, uma vez que o cuidado, sob a perspectiva dos CP, está diretamente ligado à possibilidade do sofrimento ocasionado pela doença; já para a Enfermagem, o cuidado está relacionado com a essência de sua atuação e algumas vezes não tem relação com a doença ${ }^{(2,12)}$.

No estudo, houve a expressão da fragmentação do cuidado multiprofissional, sendo a Enfermagem, em muitas situações, um elo de comunicação entre os profissionais; não há um momento em que todos discutam estratégias para atender as necessidades de cuidado do paciente e de seus familiares. Logo, é evidenciada mais a multidisciplinaridade do que a interdisciplinaridade, em que ocorre a troca de informações entre profissões que trabalham isoladamente e não a horizontalização de informações, o enriquecimento mútuo de conhecimento e a possibilidade de crescimento conjunto de todas as profissões envolvidas ${ }^{(13,14)}$.

A filosofia dos CP preconiza que haja um resgate da humanização da assistência prestada aos pacientes, focado nas angústias das dimensões físicas, psíquicas, sociais e espirituais e não na doença, tornando esse cuidado algo a ser realizado por uma equipe de profissionais e não apenas por uma profissão ${ }^{(14,15)}$.

Ainda quanto aos CP e à atuação multiprofissional, os participantes referiram que a atuação em forma de consultoria da Comissão de CP e Controle da Dor dificulta o processo de cuidar da pessoa com doença crônica, principalmente em casos mais complexos, pois a equipe que presta a consultoria não está permanentemente, e nem sempre periodicamente, avaliando o paciente.

Estudos sinalizam que as atividades na modalidade de consultoria proporcionam dificuldades na assistência, principalmente devido à ausência de vínculos entre a equipe que realiza a consultoria e a que presta o cuidado, em especial no que se refere à prescrição medicamentosa. Entretanto, para hospitais-gerais, esse tipo de organização de trabalho mostra vantagens, como baixo custo para implementação(16,17).

Ainda observou-se que a relação da Comissão de CP e Controle da Dor com os integrantes da equipe multiprofissional do cenário do estudo é prejudicada pela comunicação ineficaz, somada à carência de formação e de educação permanente. Especificamente no que se refere à filosofia dos $\mathrm{CP}$, a comunicação também se mostrou ineficaz. Assim, a comunicação torna-se um desafio diário para quem trabalha em equipe, estabelecendo o aprendizado coletivo do acolhimento de diferenças e o exercício das capacidades individuais e do respeito pelo outro ${ }^{(7,13,18,19)}$

Há que se salientar que a filosofia dos CP preconiza o princípio da busca da qualidade de vida. Esse princípio não foi mencionado pelos entrevistados, o que nos leva a inferir que a formação ainda está pautada no modelo biomédico, que tem na cura da doença seu foco, e não em como o processo de cura, ou de adoecimento, irá acontecer.

Sinaliza-se como uma limitação do estudo a seleção específica dos enfermeiros como participantes da pesquisa, na medida em que não foi possivel averiguar se os demais profissionais que formam a equipe multiprofissional da instituição possuem a mesma interpretação sobre CP. 


\section{CONCLUSÃO}

O conceito e princípios dos CP não estão inseridos de forma plena na prática dos cuidados e na compreensão dos participantes do estudo, evidenciando lacunas na formação e uma comunicação ineficaz. Há uma atuação contraditória da equipe. De um lado, ela define que o paciente esteja em CP, quando a morte é esperada para horas e/ou dias; entretanto, ainda são instituidas medidas que causam desconforto, como exames e procedimentos invasivos.

Evidencia-se como uma potencialidade deste estudo a possibilidade dos profissionais refletirem acerca do que significa implementar CP com qualidade. Uma reflexão que deve visibilizar, aos profissionais e às instituições de saúde, a demanda de um compromisso ético e de uma atuação em equipe sintonizada e com competências específicas em termos de cuidado.

\section{REFERÊNCIAS}

1. World Health Organization. Definition of Palliative Care. 2002. [Citado 2015 Jul 21]. Disponivel em: <http://www.who.int/cancer/palliative/ definition/en/>.

2. Matsumoto DY. Cuidados Paliativos: conceito, fundamentos e principios. In. Academia Nacional de Cuidados Paliativos. Manual de cuidados paliativos. POA: Sulina, 2012. 23-31.

3. Garcia JBS, Rodrigues RF, Lima SF. La estructuración de um servicio de cuidados paliativos en Brasil - relato de una experiencia. Rev Bras Anestesiol. 2014 [Citado 2014 Jul 10] 64(4): 286-91. Disponivel em: <http:// ac.els-cdn.com/S2255496313001451/1-s2.0-S2255496313001451-main. pdf?_tid=8204c8ce-2162-1le4-aa8b-00000aab0f278acdnat=1407766950 _7b3ac7545f708ecldd7060dae68c44ea>.

4. Moritz RD, Deicos A, Capalbo M, Forte DN, Kretzer LP, Logo , et al. II Fórum do "Grupo de Estudos do Fim da Vida do Cone Sul": definições, recomendações e ações integradas para cuidados paliativos na unidade de terapia intensiva de adultos e pediátrica. Rev Bras Terapia Intensiva. 2011; 23(1): 24-9.

5. Bifulco VA, Iochida, LC. Palliative care in the formation of health care professionals. Rev. Bras. Educação médica. 2009 [Citado 2014 Ago 10] 33(1): 92-100. Disponivel em: <http://www.scielo.br/pdf/rbem/v33nl/13. $\mathrm{pdf}>$.

6. Shawler C. Palliative and end-of-life care: using a Standardized Patient Family for Gerontological Nurse Practitioner Students. Nursing Education Perspectives. 2011 Maio/Jun [Citado 2014 Dez 20]; 32(3): 168-72. Disponivel em: http://dx.doi.org/10.5480/1536-5026-32.3.168.

7. Vargas MAO, Vivon J, Vieira RW, Rolim J, Ramos FRS, Ferrazzo S, et al Redefining palliative care at a specialized care center: a possible reality? Rev. Texto Contexto Enferm 2013 [Citado 2014 Ago 15]; 22(3): 637-45 Disponivel em: http://www.scielo.br/pdf/tce/v22n3/en_v22n3a09.pdf.

8 Martineli LRL, Carvalho MVB. A atuação multidisciplinar em cuidados paliativos: o lidar com crianças e adolescentes onco-hematológicos. Rev enferm UFPE on line 2011 Ago [Citado 2014 Mar 01]; 5(6):1444-51. Disponivel em: http///www.ufpe br/revistaenfermagem/indexphp/revista/issue/ view/44.

9 Kelly K, throne S, Virani R, Marray P. Ferrel B. Expanding palliative care nursing education in California: the ELNEC Geriatric project. International Journal of Palliative Nursing 2011 [Citado 2014 Jan 10]: 17(4): 188-94. Disponivel em: http://ukpmc.ac.uk/abstract/MED/21537321.

10 Weissman DE, Meier DE. Identifying Patients in Need of a Palliative Care Assessment in the Hospital Setting - A Consensus Report from the Center to Advance Palliative Care. Journal of Palliative Medicine. 2011 Jan [Citado 2012 Jan 08]; 14(1): 17-23. Disponivel em: http://online.liebertpub.com/doi/ abs/10.1089/jpm.2010.0347.
11 Parker D, Hodgkinson B. Comparison of palliative care outcome measures used to assess the quality of palliative care provided in longterm care facilities: a systematic review. Palliative Medicine. 2011 Jan [Citado 2012 Jan 10]; 25(1): 5-20. http://online.sagepub.com/search/s?su bmit=yesEsrc=hw\&andorexactfulltext=and\&fulltext=Comparison+of+pa lliative+care+outcome+measures+used+to+assess+the+quality+of+palliat ive+care+provided+in+long-term+care+facilities\%3A+a+systematic+review हx=11हy=9.

12. Durante ALTC, Tonini T, Armini LR. Confort in palliative care: the knowhow of nurses in general hospital. Rev. enferm. UFPE on-line 2014 [Citado 2014 Jul 15]; 8(3): 530-36. Disponivel em: <http://www.revista.ufpe.br/ revistaenfermagem/index.php/revista/article/download/5849/8598>

13. Silva MMS, Moreira MC, Leita JL, Erdmann AL. Nursing work at night in palliative oncology care. Rev Latino-Am Enferm. 2013 [Citado 2014 Ago 10]; 21(3). Disponivel em: <http://www.scielo.br/scielo.php?script=sci_ arttextEpid=S0104-11692013000300773>

14. Matos E, Pires DEP, Gelbcke FL. Implicações da interdisciplinaridade na organização do trabalho da enfermagem: estudo em equipe de cuidados paliativos. Rev. Eletr. Enf. 2012 [Citado 2014 Ago 10]; 14(2): 230-39. Disponivel em: <http://www.fen.ufg.br/revista/v14/n2/v14n2aO2.htm>.

15. Fernandes MA. Evangelista CB. Platel ICS. Agra G. Lopes MS. Rodrigues FA. Percepções dos enfermeiros sobre o significado dos cuidados paliativos em pacientes com câncer terminal. Rev. Ciênc. Saúde Coletiva. 2013 [Citado 2014 Jul 10]; 18(9). Disponivel em: <http://www.scielosp.org/ scielo.php?script=sci_arttext\&pid=S1413-81232013001700013>

16. Doyle D. Getting started: quidelines and suggestions for those starting a hospice/ palliative care service. Houston: IAHPC. 2009. [Citado $2014 \mathrm{Jul}$ 10]. Disponivel em: <http://hospicemadreteresa.org.ar/esp/wp-content/ uploads/2011/11/iahpc-getting-started-2nd-ed.pdf>.

17. Maciel MGS. Organização de serviços de Cuidado Paliativo. In: academia nacional de cuidados paliativos. Manual de cuidados paliativos. RJ: Diagraphic, 2009. [Citado 2014 Jul 10]. Disponivel em: <http://nhu.ufms br/Bioetica/Textos/Morte\%20e\%200\%20Morrer/MANUAL\%20DE\%20 CUIDADOS\%2OPALIATIVOS.pdf>.

18. Araújo MMT, Silva MJP. O conhecimento de estratégias de comunicação no atendimento à dimensão emocional em cuidados paliativos. Rev. Texto Contexto Enferm. 2012 [Citado 2014 Jul 10]: 21(1). Disponivel em:

<http://www.scielo.br/scielo.php?script=sci_arttext\&pid=S010407072012000100014>.

19. Cardoso DH, Muniz RM, Schwartz E, Arrieira ICO. Cuidados paliativos na assistência hospitalar: a vivência de uma equipe multiprofissional. Rev. Texto Contexto Enferm 2013 [Citado Abr 20]; 22(4): 1134-41. Disponivel em: <http://www.scielo.br/scielo.php?script=sci_arttext\&pid=SO10 4-07072013000400032>. 